\title{
Parent calcium-rich-food practices/perceptions are associated with calcium intake among parents and their early adolescent children
}

\author{
Marla Reicks 1,* Dennis Degeneffe ${ }^{2}$, Koel Ghosh², Christine Bruhn³ , L Suzanne Goodell ${ }^{4}$, \\ Carolyn Gunther ${ }^{5}$, Garry Auld 6 , Miriam Ballejos ${ }^{7}$, Carol Boushey ${ }^{8}$, , Mary Cluskey 9 , \\ Scottie Misner ${ }^{10}$, Beth Olson ${ }^{11}$, SiewSun Wong ${ }^{12}$ and Sahar Zaghloul ${ }^{13,} \ddagger$ \\ 'Department of Food Science and Nutrition, University of Minnesota, 1334 Eckles Avenue, St. Paul, MN \\ 55108 , USA: ${ }^{2}$ Department of Applied Economics, University of Minnesota, St. Paul, MN, USA: ${ }^{3}$ Department of \\ Food Science and Technology, University of California at Davis, Davis, CA, USA: ${ }^{4}$ Department of Food, \\ Bioprocessing \& Nutrition Sciences, North Carolina State University, Raleigh, NC, USA: ${ }^{5}$ Department of Human \\ Nutrition, Ohio State University, Columbus, OH, USA: ${ }^{6}$ Department of Food Science \& Human Nutrition, \\ Colorado State University, Fort Collins, CO, USA: ${ }^{7}$ Puyallup Research and Extension Center, Washington State \\ University, Puyallup, WA, USA: ${ }^{8}$ Department of Foods and Nutrition, Purdue University, West Lafayette, IN, USA: \\ ${ }^{9}$ Department of Nutrition and Exercise Science, Oregon State University, Corvallis, OR, USA: ${ }^{10}$ Department of \\ Nutritional Sciences, University of Arizona, Tucson, AZ, USA: " $D$ Department of Food Science \& Human \\ Nutrition, Michigan State University, East Lansing, MI, USA: ${ }^{2}$ Department of Nutrition and Food Science, Utah \\ State University, Logan, UT, USA: ${ }^{13}$ Department of Human Nutrition, Food and Animal Sciences, University of \\ Hawaii at Manoa, Honolulu, HI, USA
}

Submitted 1 November 2010: Accepted 14 April 2011: First published online 1 June 2011

\begin{abstract}
Objective: The study aimed to (i) segment parents of early adolescents into subgroups according to their $\mathrm{Ca}$-rich-food (CRF) practices and perceptions regarding early adolescent CRF intake and (ii) determine whether Ca intake of parents and early adolescents differed by subgroup.

Design: A cross-sectional convenience sample of 509 parents and their early adolescent children completed a questionnaire in 2006-2007 to assess parent CRF practices and perceptions and to estimate parent and child Ca intakes.

Setting: Self-administered questionnaires were completed in community settings or homes across nine US states.

Subjects: Parents self-reporting as Asian, Hispanic or non-Hispanic White with a child aged 10-13 years were recruited through youth or parent events.

Results: Three parent CRF practice/perception segments were identified, including 'Dedicated-Milk Providers/Drinkers' (49\%), 'Water Regulars' (30\%) and 'Sweet-Drink-Permissive Parents' (23\%). Dedicated-Milk Providers/Drinkers were somewhat older and more likely to be non-Hispanic White than other groups. Ca intakes from all food sources, milk/dairy foods and milk only, and milk intakes, were higher among early adolescent children of Dedicated-Milk Providers/ Drinkers compared with early adolescents of parents in other segments. Soda pop intakes were highest for early adolescents with parents in the Water Regulars group than other groups. Dedicated-Milk Providers/Drinkers scored higher on culture/tradition, health benefits and ease of use/convenience subscales and lower on a dairy/milk intolerance subscale and were more likely to report eating family dinners daily than parents in the other groups.

Conclusions: Parent education programmes should address CRF practices/perceptions tailored to parent group to improve Ca intake of early adolescent children.
\end{abstract}

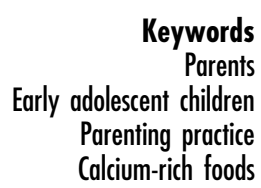

eywords Calcium-rich foods $\dagger$ Current address: Epidemiology Program, University of Hawaii Cancer Center, Honolulu, HI, USA.

¥ Current address: National Nutrition Institute, Cairo, Egypt.
Only $15 \%$ of girls and $23 \%$ of boys (9-13 years) met recommendations for $\mathrm{Ca}$ intake from all food sources based on US national dietary intake data $(2005-2006)^{(1)}$. Early adolescence is a critical time in physical development, 
when adequate levels of $\mathrm{Ca}$ can optimize bone mineralization $^{(2,3)}$. The frequency of family meals and availability of milk at meals have been positively associated with intake of Ca-rich foods (CRF) by adolescents, while soft drinks consumption has been negatively associated ${ }^{(4,5)}$. Making CRF accessible and role modelling were suggested as strategies that mothers could use to improve $\mathrm{Ca}$ intake of adolescent children $^{(6)}$. Others have shown that when mothers drank milk more frequently and served milk to younger girls (5-9 years), girls were more likely to meet Ca recommendations ${ }^{(7)}$. Familial correlations of $\mathrm{Ca}$ intakes have also been observed between adolescent daughters and their mothers ${ }^{(8,9)}$.

Social Cognitive Theory (SCT) proposes that individual, behavioural and socio-environmental factors interrelate to influence behaviour $^{(10)}$. Consistent with SCT, data indicate that $\mathrm{Ca}$ intake among adults is mediated by numerous factors on multiple levels (personal, behavioural, socioenvironmental). Individual factors that may directly or indirectly influence $\mathrm{Ca}$ intake of parents include perceptions of health benefits or concern about the adequacy of $\mathrm{Ca}$ intake $^{(11)}$, knowledge of Ca sources and concerns about weight gain from eating $\mathrm{CRF}^{(12,13)}$, and dairy or milk intolerance $^{(14)}$. Behavioural factors, such as eating food away from home, may also play an important role. Food prepared and consumed away from home tends to contain less dietary $\mathrm{Ca}$ per kilojoule compared with food prepared and consumed at home ${ }^{(15)}$. Unfortunately, lack of time and work stress can lead parents to eat more meals away from home rather than at home ${ }^{(16)}$. Socio-environmental factors which may influence $\mathrm{Ca}$ intake among early adolescents include parenting practices/perceptions that encourage intake of $\mathrm{CRF}^{(17)}$ such as role modelling, making CRF available in the home and perceptions that dairy foods are important for health.

Data suggest that race/ethnicity factors may affect choice of CRF and therefore a parent's tendency to encourage or model intake for children. For example, most husbands/ male partners of women of Chinese ethnicity living in the USA preferred a traditional Chinese diet (90\%) and in some households (19\%) exerted a strong influence on dietary intake of the whole family ${ }^{(18)}$. Similarly, Ca intake from milk was negatively associated with being an Asian or Hispanic parent compared with reference non-Hispanic White parents and with higher dairy/milk intolerance scores ${ }^{(17)}$. This finding is consistent with reports indicating a high incidence of dairy/milk intolerance in Asian and Hispanic groups ${ }^{(19)}$.

Tailored nutrition education has the potential to be more effective than non-tailored education according to recent reviews ${ }^{(20,21)}$. Segmentation analysis is a method used to tailor education programmes according to subgroup characteristics by identifying audience groups sharing similar attitudes about food and nutrition, lifestyle and physical characteristics $^{(22-26)}$. Importantly, segmentation analysis was used in previous studies to subdivide groups of parents according to the use of diverse parenting practices that facilitate fruit and vegetable consumption among pre-school children $^{(26)}$ and by psychographic factors that influence mothers' food decisions ${ }^{(27)}$. While this approach holds promise for improving parent nutrition education, studies of this nature are limited, especially regarding parent CRF practices and perceptions. Therefore, the purpose of the present study was to (i) segment a convenience sample of Asian, Hispanic and non-Hispanic White parents of early adolescents based on similarities of reported CRF practices and perceptions and (ii) determine how early adolescent and parent $\mathrm{Ca}$ intake differs according to parent segment.

\section{Experimental methods}

\section{Study design and sample recruitment}

The present cross-sectional study was part of a larger study of parent and household influences on $\mathrm{Ca}$ intake of early adolescents and their parents in the USA ${ }^{(17)}$. Separate questionnaires were completed once by a convenience sample of children aged 10-13 years and an adult responsible for food acquisition and preparation in the household. For $98 \%$ of the adult questionnaires, the respondent was the child's parent; therefore, the adult respondent is referred to as 'parent' throughout the current report. Inclusion criteria for parents included: (i) having lived in the USA for at least 12 months; (ii) able to read/ speak English; and (iii) self-identifying as non-Hispanic White, Hispanic or Latino, or Asian or Asian American, or a mixture of any of these three groups.

Participants were recruited using fliers, verbal announcements, written announcements in bulletins or newsletters, personal contacts and presentations at group events. Organizations and groups involved in the present study included US Cooperative Extension Service (e.g. US national nutrition education programmes for low-income audiences such as the Expanded Food Nutrition Education Program and the Supplemental Nutrition Assistance Program Education for individuals receiving food benefits, 4-H youth programmes), faith-based groups, afterschool programmes, sports teams, scouting groups and adult groups. These organizations and groups serve a wide variety of participants, thus assuring diversity of the study sample regarding education and income. Recruitment information was either distributed directly to parents while they were attending adult or child events/ programmes or to children while they were attending events/programmes with a request that they provide the information to parents. A total of 633 matched parent/ child pairs from nine states in the USA (Arizona, California, Colorado, Hawaii, Michigan, Minnesota, Oregon, Utah and Washington) completed paper-based questionnaires in 2006-2007, with complete data available for all segmentation variables from 509 parents.

The study protocols were approved by the Institutional Review Board of all participating universities. Each parent and child provided written informed consent and assent, respectively. 


\section{Data collection}

A standardized data collection protocol was developed and used to ensure that self-administered questionnaire data were collected in a consistent manner across sites. Researchers met with parents and children to distribute questionnaires in the home or in community settings (community centres, libraries, athletic facilities, etc.). In four states, all questionnaires were distributed by researchers in person only; however, in five states researchers also used mailed packets to collect completed questionnaires from $14 \%$ to $50 \%$ of parents in their respective states. Parents took an average of $30 \mathrm{~min}$ and children approximately 20-30 min to complete the questionnaire. In return for participation, parents and children were each given cash, gift certificates/cards or merchandise (ranging in value from \$US 5 to \$US 20) per each institution's remuneration guidelines. All questionnaires were completed in English.

\section{Parent and child questionnaires}

In-depth interviews were previously conducted with nonHispanic White, Hispanic and Asian parents of early adolescent children to assess knowledge on $\mathrm{Ca}^{(28)}$ and parenting practices and meal patterns regarding CRF intake by children $^{(12,29)}$. Findings were used to develop items for a parent questionnaire based on two constructs: (i) parental practices and perceptions regarding child intake of CRF; and (ii) attitudes/preferences regarding parental intake of CRF.

Items were tested for clarity and understanding and revised as needed based on results from individual cognitive interviews with thirty-two parents (nine Asian, ten Hispanic and thirteen non-Hispanic White) across seven states. Fourteen subscales were constructed describing the two constructs. The subscales met standards for psychometric properties, with modest to acceptable Cronbach $\alpha$ coefficients for internal consistency $(0 \cdot 50-0 \cdot 79)$ and Pearson correlation test-retest reliability coefficients ranging from 0.68 to $0.85^{(17)}$. The child questionnaire included items assessing psychosocial factors associated with $\mathrm{Ca}$ intake (results not reported here) and Ca intake via a Ca-specific $\mathrm{FFQ}^{(30)}$.

\section{Measurements and variables}

Six of the fourteen subscales were based on fifty-three parent questionnaire items describing parenting practices and perceptions regarding child intake of CRF, including: (i) making CRF available (ten items, $\alpha=0 \cdot 71$ ); (ii) encouraging intake of healthy foods (five items, $\alpha=0 \cdot 75$ ); (iii) having healthy beverage expectations (thirteen items, $\alpha=0.79$ ); (iv) perceptions of the importance of Ca sources (seven items, $\alpha=0 \cdot 65$ ); (v) role modelling (fifteen items, $\alpha=0 \cdot 70$ ); and (vi) the nature of family meals in the household (three items, $\alpha=0 \cdot 68$ ). These fifty-three items were used as segmentation variables in the cluster analysis.

The remaining eight subscales were based on parent questionnaire items which assessed parent attitudes and preferences regarding parental intake of CRF, including: (i) culture/tradition (five items, $\alpha=0.50$ ); (ii) concern about cost (five items, $\alpha=0 \cdot 57$ ); (iii) concern about weight (four items, $\alpha=0 \cdot 60$ ); (iv) concern about adequacy of parents' own intake (three items, $\alpha=0.73$ ); (v) liking/taste (seven items, $\alpha=0.60$ ); (vi) health benefits (ten items, $\alpha=0.60$ ); (vii) dairy/milk intolerance (four items, $\alpha=$ 0.62 ); and (viii) ease of use/convenience (four items, $\alpha=0 \cdot 67$ ). Parent knowledge regarding Ca requirements and sources was assessed as a score based on correct responses to seven knowledge items. The frequency of eating food away from home and eating meals together was also assessed. Differences in attitudes/preferences and knowledge based on these subscales and the knowledge score were determined for parent segments.

$\mathrm{Ca}$ intakes (estimated $\mathrm{mg} / \mathrm{d}$ ) for both parents and early adolescents were measured using a semi-quantitative Ca-specific FFQ (seventy-nine items or groups of items) developed and evaluated for use among non-Hispanic White, Hispanic and Asian youth ${ }^{(30)}$. Major food groups included beverages; dairy products; combination foods; vegetables, grains and nuts; seafood; and other foods. For each food item, a commonly used portion size was listed with a question asking how often the food item was consumed during the past month. The number of frequency responses ran from four to seven, ranging from 'Never or less than once per month' to 'Four or more servings per day'. A Ca value was assigned to each food item on the FFQ based on the frequency of consumption, serving size and $\mathrm{Ca}$ content of the food item. The instrument performed well when used with adult women, as estimates for $\mathrm{Ca}$ intake were comparable to those obtained from $2 \mathrm{~d}$ food records $(r=0.52)^{(31)}$. Therefore, the same FFQ was used with parents and children to allow for comparison of $\mathrm{Ca}$ intakes between them. Demographic information about the parent, spouse and the 10-13-year-old child was also collected.

\section{Data analysis}

For classification to a race/ethnic group, participants were first asked to mark whether they were Hispanic or not Hispanic. Next they were asked which racial groups(s) they considered themselves to be, using as choices American Indian or Alaska Native, Asian, Black or African American, White or Caucasian, and other. Participants who selected more than one group were classified as Asian, Hispanic or non-Hispanic White if one of these groups was selected. Those who chose Asian and nonHispanic White, or Hispanic and non-Hispanic White, were classified as Asian or Hispanic, respectively. In instances where Asian and Hispanic were both selected ( $n$ 3), the predominant ethnic group of the sampling location was used for classification as either Asian or Hispanic.

Segmentation variables included the fifty-three individual items which made up the six CRF parenting practices and perceptions subscales regarding CRF intake of early 
adolescent children. Although all segmentation variables were measured on a 5-point scale, all variables were standardized by the variable mean and standard deviation. Scales for some variables were reverse coded, so all items reflected a positive response prior to cluster analysis.

JMP software version 8 (SAS Institute Inc., Cary, NC, USA) was used to form clusters (segments) which were internally homogeneous but different from each other. The $k$-means approach to clustering was used to determine the final clusters ${ }^{(32)}$. First, the number of clusters $(k)$ to be determined was specified in JMP. Next, a search algorithm was used to find $k$ points (or parents) in the data (seeds) that were not close to each other. Each seed was then treated as a cluster centre. The iterative routine assigned each point to the closest cluster. For each cluster, a new cluster centre was formed as the means (centroid) of the points currently in the cluster. This process alternated between assigning points to clusters and recalculating cluster centres until the clusters became stable.

Four cluster solutions were produced yielding three, four, five and six clusters (i.e. $k=3,4,5,6$ ). Each cluster solution was studied with respect to responses to the segmentation variables used in the cluster analysis. Indices were used to compare responses from groups of parents belonging to one of the resulting clusters in that cluster solution relative to the total sample of respondents. These indices were based on the percentage of respondents who selected the top two points of the rating scales (percentage of the segment indicating either a ' 4 ' or a ' 5 ' on the 5-point scales divided by the percentage of the total sample selecting the top two scale points) ${ }^{(33)}$. After considering all four cluster solutions, the three-cluster solution was selected because (i) it was judged to be the most meaningfully different across segments, (ii) the distance to the nearest cluster values indicated a reasonable separation between cluster centroids and (iii) it resulted in adequate sample sizes for further analysis of study results. The three clusters were given descriptive names and described according to attitude and behavioural patterns that distinguished them from one another according to the calculated indices. In analysing the clusters indices, those greater than $1 \cdot 20$ and less than $0 \cdot 80$ were considered higher and lower, respectively, than the average for the total sample. The description of each cluster reflected a pattern of higher- or lower-than-average indices across multiple measures. For example, cluster 2 yielded indices of 1.82 for drinking milk at every meal, 1.70 for drinking milk with lunch, 1.67 for drinking milk with snacks, 1.33 for keeping yoghurt in the home for children, etc., reflecting a clear theme around affinity for milk/dairy products. Based on this pattern, cluster 2 was named 'Dedicated-Milk Providers/Drinkers'.

Tests to assess differences in demographic characteristics, food and nutrient intakes, and behavioural factors between clusters were conducted using the SAS statistical software package version 9.2 (SAS Institute Inc., Cary,
NC, USA). Data from parents and early adolescents whose Ca intakes were outside established cut-off points $(<100$ or $>2500 \mathrm{mg} / \mathrm{d}^{(34)}$; eleven parents and twenty-two early adolescents) were excluded from further analysis regarding $\mathrm{Ca}$ and beverage intakes. Descriptive statistics (mean and standard deviation) were used to examine daily intake of Ca among parents and early adolescents from all food sources, dairy foods, and milk and beverage consumption by parent CRF practice/perception segments or groups. ANOVA was performed to characterize the sample across parent CRF practice/perception segments for quantitative variables (parent and child $\mathrm{Ca}$ and beverage intakes, parent scores on practice, attitude/perception subscales and eating away from home and eating meals together). This was followed by Duncan's multiple-range test for post hoc analysis. For categorical variables (e.g. demographic and household factors), $\chi^{2}$ tests were used.

\section{Results}

Most respondents were mothers (89\%) from homes with two adults present (70\%), and $53 \%$ of the early adolescents were girls. Most parents were between 31 and 50 years of age $(88 \%)$ and had attended some college or had a 4-year degree (75\%; Table 1). Half self-reported as Asian (17\%) or Hispanic (32\%), and half as non-Hispanic White (47\%). About $10 \%$ of Asian and Hispanic parents indicated they did not speak English at home. A quarter (25\%) participated in a US federal nutrition assistance programme, and about $75 \%$ were employed full- or part-time.

The three parent CRF practice/perception segments were named and described according to attitude and behavioural patterns that distinguished them from one another according to the indices described previously. The first segment was named 'Sweet-Drink-Permissive Parents'. These parents reported allowing their child to drink fruit drinks (e.g. Kool Aid ${ }^{\circledR}$, Sunny Delight ${ }^{\circledR}$ ) and regular soda pop, although they also reported being unlikely to keep these beverages in their homes. They were not likely to report trying to get their child to drink milk with meals or snacks. They were also not apt to report that they provided plain or flavoured milk for their child, or drank milk themselves at meals, for snacks or away from home. They placed a high importance on nondairy foods like dark green leafy vegetables and tofu to help their child get the Ca needed. The second segment was named 'Dedicated-Milk Providers/Drinkers'. These parents tended to report a high frequency of drinking milk themselves and providing it to their child across meal occasions and snacks, both at home and away from home. They also were likely to report both providing and eating yoghurt and serving macaroni and cheese to their child. These parents were not likely to report that they allowed their child to have fruit drinks (e.g. Kool Aid, Sunny Delight) or to have these beverages in the home. 
Table 1 Demographic characteristics of parents by parent CRF practice/perception segment; convenience sample of parents with a 10-13year-old child from nine states in the USA (Arizona, California, Colorado, Hawaii, Michigan, Minnesota, Oregon, Utah and Washington), 2006-2007

\begin{tabular}{|c|c|c|c|c|c|c|c|c|c|}
\hline & \multicolumn{2}{|c|}{$\begin{array}{c}\text { All } \\
\text { parents }\end{array}$} & \multicolumn{2}{|c|}{$\begin{array}{c}\text { Sweet-Drink-Permissive } \\
\text { Parents }\end{array}$} & \multicolumn{2}{|c|}{$\begin{array}{l}\text { Dedicated-Milk } \\
\text { Providers/Drinkers }\end{array}$} & \multicolumn{2}{|c|}{$\begin{array}{l}\text { Water } \\
\text { Regulars }\end{array}$} & \multirow{2}{*}{$\begin{array}{l}P \text { value } \\
\left(\chi^{2} \text { test }\right)\end{array}$} \\
\hline & $\%$ & $n$ & $\%$ & $n$ & $\%$ & $n$ & $\%$ & $n$ & \\
\hline Age (years) & - & 507 & - & 113 & - & 243 & - & 151 & $<0.0001$ \\
\hline $18-30$ & $6 \cdot 4$ & 31 & $7 \cdot 1$ & 8 & $6 \cdot 2$ & 15 & $5 \cdot 3$ & 8 & \\
\hline $31-40$ & $42 \cdot 4$ & 215 & $39 \cdot 8$ & 45 & $32 \cdot 9$ & 80 & $59 \cdot 6$ & 90 & \\
\hline $41-50$ & $45 \cdot 0$ & 228 & $46 \cdot 0$ & 52 & $54 \cdot 3$ & 132 & $29 \cdot 1$ & 44 & \\
\hline $51+$ & $6 \cdot 5$ & 33 & $7 \cdot 1$ & 8 & $6 \cdot 6$ & 16 & $6 \cdot 0$ & 9 & \\
\hline Education & - & 504 & - & 112 & - & 241 & - & 151 & $<0.0001$ \\
\hline$<$ High school & $9 \cdot 1$ & 46 & $7 \cdot 1$ & 8 & $7 \cdot 1$ & 17 & $13 \cdot 9$ & 21 & \\
\hline High school & $16 \cdot 3$ & 82 & $7 \cdot 1$ & 8 & $12 \cdot 0$ & 29 & $29 \cdot 8$ & 45 & \\
\hline Some college & $35 \cdot 1$ & 177 & $40 \cdot 2$ & 45 & $29 \cdot 5$ & 71 & $40 \cdot 4$ & 61 & \\
\hline 4-year degree & $39 \cdot 5$ & 199 & $45 \cdot 5$ & 51 & $51 \cdot 5$ & 124 & $15 \cdot 9$ & 24 & \\
\hline Race/ethnic group & - & 509 & - & 115 & - & 243 & - & 151 & $<0.0001$ \\
\hline Asian & $16 \cdot 9$ & 86 & $29 \cdot 6$ & 34 & $15 \cdot 6$ & 38 & $9 \cdot 3$ & 14 & \\
\hline Hispanic & $32 \cdot 2$ & 164 & $27 \cdot 0$ & 31 & $22 \cdot 2$ & 54 & $52 \cdot 3$ & 79 & \\
\hline Non Hispanic White & $47 \cdot 4$ & 241 & $37 \cdot 4$ & 43 & $58 \cdot 4$ & 142 & $37 \cdot 1$ & 56 & \\
\hline Other & $3 \cdot 5$ & 18 & $6 \cdot 1$ & 7 & $3 \cdot 7$ & 9 & $1 \cdot 3$ & 2 & \\
\hline Race/ethnic group of spouse & - & 428 & - & 98 & - & 212 & - & 118 & $<0.0001$ \\
\hline Asian & $15 \cdot 4$ & 66 & $25 \cdot 5$ & 25 & $15 \cdot 1$ & 32 & $7 \cdot 6$ & 9 & \\
\hline Hispanic & $30 \cdot 1$ & 129 & $21 \cdot 4$ & 21 & $21 \cdot 7$ & 46 & $52 \cdot 5$ & 62 & \\
\hline Non-Hispanic White & $48 \cdot 1$ & 206 & $44 \cdot 9$ & 44 & $57 \cdot 1$ & 121 & $34 \cdot 8$ & 41 & \\
\hline Other & $6 \cdot 3$ & 27 & $8 \cdot 2$ & 8 & $6 \cdot 1$ & 13 & $5 \cdot 1$ & 6 & \\
\hline Participation in assistance programmes & - & 509 & - & 113 & - & 241 & - & 150 & $<0.0001$ \\
\hline No assistance & $74 \cdot 7$ & 380 & $79 \cdot 7$ & 90 & $82 \cdot 6$ & 199 & $60 \cdot 7$ & 91 & \\
\hline One or more programmes & $25 \cdot 3$ & 129 & $20 \cdot 4$ & 23 & $17 \cdot 4$ & 42 & $39 \cdot 3$ & 59 & \\
\hline Language spoken at home & - & 448 & - & 104 & - & 202 & - & 142 & $<0.0001$ \\
\hline No English & $8 \cdot 5$ & 38 & $9 \cdot 6$ & 10 & $7 \cdot 4$ & 15 & $9 \cdot 2$ & 13 & \\
\hline Another language more than English & $8 \cdot 9$ & 40 & $5 \cdot 8$ & 6 & $10 \cdot 9$ & 22 & $8 \cdot \overline{5}$ & 12 & \\
\hline Another language and English equally & $12 \cdot 5$ & 56 & $8 \cdot 7$ & 9 & $5 \cdot 5$ & 11 & $25 \cdot 4$ & 36 & \\
\hline English more than another language & $11 \cdot 6$ & 52 & $15 \cdot 4$ & 16 & $10 \cdot 9$ & 22 & $9 \cdot 9$ & 14 & \\
\hline English only & $58 \cdot 5$ & 262 & $60 \cdot 6$ & 63 & $65 \cdot 4$ & 132 & $47 \cdot 2$ & 67 & \\
\hline
\end{tabular}

CRF, Ca-rich food(s).

Parents in this segment reported often trying to get their child to drink milk for meals, snacks and away from home. They considered dairy foods important sources of Ca for their child. The third segment was named 'Water Regulars'. They reported a high frequency of drinking water with meals and snacks themselves, and encouraging their child to drink water at meals. However, they also were likely to report making fruit drinks (e.g. Kool Aid, Sunny Delight), soda pop, chocolate milk and juice with added $\mathrm{Ca}$ available in the home.

Demographic characteristics differed somewhat by parent CRF practice/perception segment. Parents in the first segment were similar to the total sample with respect to age, tended to be slightly more educated with $86 \%$ having had some college or more, and were more likely to be Asian compared with other groups (Table 1). Parents in the second segment tended to be somewhat older than the other segments, with $54 \%$ between the ages of 41 and 50 years. They were the most educated, with $52 \%$ having a 4-year degree or more, and had the highest likelihood of being White and only speaking English at home compared with the other two groups. The third segment included parents who tended to be the youngest of all segments, with $60 \%$ aged $31-40$ years; the least educated, with $44 \%$ having a high school diploma or less; more likely to be Hispanic; less likely to only speak English at home; and more likely to participate in federal food assistance programmes compared with parents in the other two groups.

Ca intakes from all food sources, dairy foods and milk were higher in early adolescent children of DedicatedMilk Providers/Drinkers, compared with early adolescent children of Sweet-Drink-Permissive Parents or Water Regulars $(P<0 \cdot 0001$; Table 2$)$. Among parents, Ca intake from all food sources, dairy foods and milk was greater in the Dedicated-Milk Providers/Drinkers compared with the other two groups $(P<0 \cdot 0001)$. Soda pop intakes were highest for parents in the Water Regulars group and their early adolescents compared with the other groups ( $P<0.001$ and $P<0.0001$, respectively), while milk consumption was highest for parents in the DedicatedMilk Providers/Drinkers group and their early adolescents compared with the other groups $(P<0 \cdot 0001)$.

Dedicated-Milk Providers/Drinkers scored higher on the culture/tradition $(P<0 \cdot 0001)$, health benefits $(P<0 \cdot 0001)$ and ease of use/convenience subscales $(P<0 \cdot 0001)$ and lower on the dairy/milk intolerance subscale $(P<0 \cdot 0001)$ compared with parents in the other two groups (Table 3 ). 
Table 2 Calcium (mg/d) and beverage intakes (oz/d) among early adolescent children and parents by parent CRF practice/perception segment; convenience sample of parents with a 10-13-year-old child from nine states in the USA (Arizona, California, Colorado, Hawaii, Michigan, Minnesota, Oregon, Utah and Washington), $2006-2007$

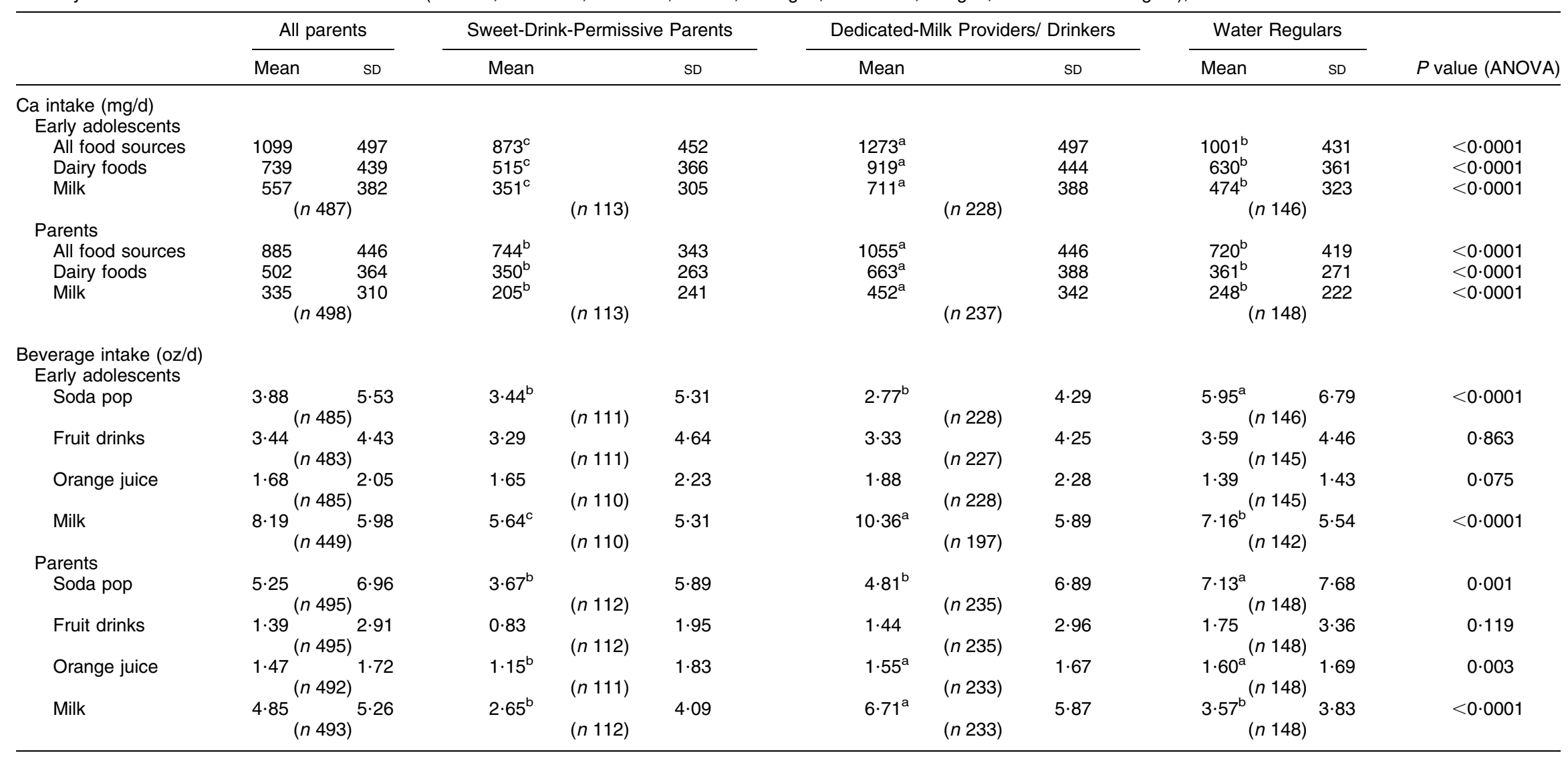

CRF, Ca-rich food(s). 
Table 3 Parent attitude and preferences regarding CRF by parent CRF practice/perception segment; convenience sample of parents with a 10-13-year-old child from nine states in the USA (Arizona, California, Colorado, Hawaii, Michigan, Minnesota, Oregon, Utah and Washington), 2006-2007

\begin{tabular}{|c|c|c|c|c|c|c|c|}
\hline & All parents & \multicolumn{2}{|c|}{$\begin{array}{l}\text { Sweet-Drink-Permissive } \\
\text { Parents }\end{array}$} & \multicolumn{2}{|c|}{$\begin{array}{l}\text { Dedicated-Milk } \\
\text { Providers/ Drinkers }\end{array}$} & $\begin{array}{l}\text { Water } \\
\text { Regulars }\end{array}$ & \multirow{2}{*}{$\begin{array}{l}P \text { value } \\
\text { (ANOVA) }\end{array}$} \\
\hline & Mean & Mean & SD & Mean & $\mathrm{SD}$ & Mean SD & \\
\hline \multicolumn{8}{|c|}{$\begin{array}{l}\text { Parent attitudes and preferences regarding } \\
\text { parent intake of CRF }\end{array}$} \\
\hline Culture/tradition & $\begin{array}{l}3 \cdot 5 \quad 0.6 \\
(n 502)\end{array}$ & $3 \cdot 3^{b}(n 111)$ & $0 \cdot 7$ & $\begin{array}{l}3 \cdot 6^{\mathrm{a}} \\
(n 241)\end{array}$ & 0.6 & $\begin{array}{l}3 \cdot 4^{\mathrm{b}} \quad 0.5 \\
(n 150)\end{array}$ & $<0.0001$ \\
\hline Concern about cost & $\begin{array}{l}3 \cdot 1 \quad 0 \cdot 7 \\
(n 480)\end{array}$ & $3 \cdot 0^{\mathrm{b}}(n 109)$ & 0.6 & $3 \cdot 0^{\mathrm{b}}(n 236)$ & 0.6 & $\begin{array}{c}3 \cdot 3^{\mathrm{a}} 0 \cdot 8 \\
(n 135)\end{array}$ & $<0.0001$ \\
\hline Liking/taste & $\begin{array}{c}3 \cdot 5 \quad 0.6 \\
(n 485)\end{array}$ & $3 \cdot 7^{\mathrm{a}}(n 114)$ & 0.6 & $3 \cdot 6^{a}(n 237)$ & 0.6 & $\begin{array}{c}3 \cdot 3^{b} \quad 0.5 \\
(n 134)\end{array}$ & $<0.0001$ \\
\hline Dairy/milk intolerance & $\begin{array}{c}1 \cdot 90 \cdot 7 \\
(n 499)\end{array}$ & $2 \cdot 1^{\mathrm{a}}(n 108)$ & $0 \cdot 8$ & $1 \cdot 7^{b}(n 238)$ & 0.7 & $\begin{array}{c}2 \cdot 0^{\mathrm{a}} \quad 0 \cdot 7 \\
(n 149)\end{array}$ & $<0.0001$ \\
\hline Health benefits & $\begin{array}{l}4 \cdot 0 \quad 0.5 \\
(n 501)\end{array}$ & $3 \cdot 8^{b}(n 113)$ & 0.5 & $4 \cdot 3^{a}(n 241)$ & $0 \cdot 4$ & $\begin{array}{c}3 \cdot 8^{\mathrm{b}} \quad 0 \cdot 4 \\
(n 147)\end{array}$ & $<0.0001$ \\
\hline Concern about weight & $\begin{array}{c}3 \cdot 6 \quad 0 \cdot 6 \\
(n 486)\end{array}$ & $3 \cdot 6$ (n 107) & 0.6 & $3 \cdot 6(n 234)$ & 0.5 & $\begin{array}{c}3.7 \quad 0.6 \\
(n 145)\end{array}$ & 0.082 \\
\hline Concern about adequacy of intake & $\begin{array}{c}3 \cdot 4 \quad 0 \cdot 7 \\
(n 499)\end{array}$ & $3.5(n 111)$ & 0.7 & $3 \cdot 4(n 241)$ & $0 \cdot 7$ & $\begin{array}{c}3 \cdot 4 \quad 0 \cdot 7 \\
(n 147)\end{array}$ & $0 \cdot 730$ \\
\hline Ease of use/convenience & $\begin{array}{c}4 \cdot 2 \quad 0 \cdot 6 \\
(n 506)\end{array}$ & $4 \cdot 1^{\mathrm{b}}(n 113)$ & 0.5 & $\begin{array}{r}4 \cdot 4^{a} \\
\quad(n 242)\end{array}$ & $0 \cdot 4$ & $\begin{array}{cc}3 \cdot 8^{\mathrm{c}} & 0 \cdot 7 \\
(n 151)\end{array}$ & $<0.0001$ \\
\hline Parent Ca knowledge score ${ }^{*}$ & $\begin{array}{l}4 \cdot 1 \quad 1 \cdot 6 \\
(n \text { 499) }\end{array}$ & $3 \cdot 5^{\mathrm{b}}(n 112)$ & $1 \cdot 6$ & $\begin{array}{l}4 \cdot 3^{\mathrm{a}} \\
\quad(n 239)\end{array}$ & $1 \cdot 4$ & $\begin{array}{c}4 \cdot 0^{\mathrm{a}, \mathrm{b}} \quad 1 \cdot 6 \\
(n 148)\end{array}$ & 0.001 \\
\hline
\end{tabular}

CRF, Ca-rich food(s).

a,b,c Mean values within a row with unlike superscript letters were significantly different ( $P$ value shown).

${ }^{*}$ Possible scores ranged from $0=$ no correct items to $7=$ all correct items.

Water Regulars scored higher on the concern about cost subscale $(P<0 \cdot 0001)$ and lower on the liking/taste subscale $(P<0 \cdot 0001)$ compared with the other two groups. No differences were observed between groups regarding measures of concern about weight or adequacy of parents' own intake.

Parents in the Water Regulars segment were more likely than other parents to report a greater frequency of eating food away from home or food prepared away from home $(P=0.039$; Table 4). No differences were observed in the number of parents who reported eating breakfast together by parent CRF practice/perception segment. However, parents in the Dedicated-Milk Providers/Drinkers group were somewhat more likely to report eating dinner together daily with other family members compared with parents in the other two groups $(P=0 \cdot 015)$.

\section{Discussion}

The segmentation variables with greatest impact on the composition of the three parent segments were based on behavioural and socio-environmental factors including beverage consumption, providing beverages for children and encouraging consumption of particular beverages. Overall, segment differences in parent and early adolescent $\mathrm{Ca}$ and beverage intakes provided evidence to support the validity of the three-segment solution. Segments also differed in individual factors, such as parent attitudes and preferences, which were consistent with the descriptions of parent segments and differences in $\mathrm{Ca}$ and beverage intakes. The results indicate that there are relevant subgroups of parents with potentially unique needs for education regarding beverage consumption among their early adolescent children. The results also indicate that application of SCT may be useful in the development of education for relevant subgroups of parents by addressing behavioural, individual and socio-environmental factors.

Beverage intake plays a prominent role in determining $\mathrm{Ca}$ intake for children and adolescents. According to 2005-2006 National Health and Nutrition Examination Survey data, about $34 \%$ of Ca intake among US children and adolescents was provided by reduced-fat, whole or skimmed milk ${ }^{(35)}$. Sweetened drinks consumption has also been associated with reduced milk and $\mathrm{Ca}$ intakes in children and adolescents ${ }^{(36-38)}$. Therefore, parental influence regarding beverage intake was expected to be associated with milk and $\mathrm{Ca}$ intakes, as was observed in the current study.

Contrary to expectation, the suggested permissive attitudes of parents in the Sweet-Drink-Permissive group did not result in the highest actual consumption of soda pop and fruit drinks by early adolescents across the segments. This may be explained in part by a lower likelihood of having these beverages available in the home for children to consume. It is also possible that children may be drinking these beverages in such large quantities that they have difficulty accurately estimating 
Table 4 Family behavioural factors by parent CRF practice/perception segment; convenience sample of parents with a 10-13-year-old child from nine states in the USA (Arizona, California, Colorado, Hawaii, Michigan, Minnesota, Oregon, Utah and Washington), 2006-2007

\begin{tabular}{|c|c|c|c|c|c|c|c|c|c|}
\hline & \multicolumn{2}{|c|}{$\begin{array}{c}\text { All } \\
\text { parents }\end{array}$} & \multicolumn{2}{|c|}{$\begin{array}{l}\text { Sweet-Drink-Permissive } \\
\text { Parents }\end{array}$} & \multicolumn{2}{|c|}{$\begin{array}{l}\text { Dedicated-Milk } \\
\text { Providers/ Drinkers }\end{array}$} & \multicolumn{2}{|c|}{$\begin{array}{l}\text { Water } \\
\text { Regulars }\end{array}$} & \multirow{2}{*}{$\begin{array}{l}P \text { value } \\
\left(\chi^{2} \text { test }\right)\end{array}$} \\
\hline & $\%$ & $n$ & $\%$ & $n$ & $\%$ & $n$ & $\%$ & $n$ & \\
\hline $\begin{array}{l}\text { Eating food away from home/food } \\
\text { prepared away from home }\end{array}$ & - & 475 & - & 106 & - & 230 & - & 139 & 0.039 \\
\hline Once every $2-3$ months & $12 \cdot 0$ & 57 & $9 \cdot 4$ & 10 & $12 \cdot 2$ & 28 & $13 \cdot 7$ & 19 & \\
\hline 1-3 times/ month & $40 \cdot 2$ & 191 & $44 \cdot 3$ & 47 & $44 \cdot 4$ & 102 & $30 \cdot 2$ & 42 & \\
\hline 1-2 times/week & $37 \cdot 9$ & 180 & $37 \cdot 7$ & 40 & $37 \cdot 0$ & 85 & $39 \cdot 6$ & 55 & \\
\hline 3-4 times/week & $8 \cdot 8$ & 42 & $8 \cdot 5$ & 9 & $5 \cdot 7$ & 13 & $14 \cdot 4$ & 20 & \\
\hline 5 or more times/week & $1 \cdot 1$ & 5 & $0 \cdot 0$ & 0 & 0.9 & 2 & $2 \cdot 3$ & 3 & \\
\hline \multicolumn{10}{|l|}{ Eating meals together } \\
\hline Breakfast & - & 506 & - & 114 & - & 242 & - & 150 & $0 \cdot 104$ \\
\hline Never & $17 \cdot 6$ & 89 & $26 \cdot 3$ & 30 & $12 \cdot 7$ & 31 & $18 \cdot 7$ & 28 & \\
\hline $1-2 \mathrm{~d} /$ week & $31 \cdot 6$ & 160 & $26 \cdot 3$ & 30 & $34 \cdot 7$ & 84 & $30 \cdot 7$ & 46 & \\
\hline $3-4 \mathrm{~d} /$ week & $19 \cdot 0$ & 96 & $15 \cdot 8$ & 18 & $19 \cdot 8$ & 48 & $20 \cdot 0$ & 30 & \\
\hline $5-6 \mathrm{~d} /$ week & $15 \cdot 6$ & 79 & $16 \cdot 7$ & 19 & $14 \cdot 1$ & 34 & $17 \cdot 3$ & 26 & \\
\hline Every day & $16 \cdot 2$ & 82 & $14 \cdot 9$ & 17 & $18 \cdot 6$ & 45 & $13 \cdot 3$ & 20 & \\
\hline Lunch & - & 508 & - & 115 & - & 243 & - & 150 & 0.032 \\
\hline Never & $16 \cdot 1$ & 82 & $22 \cdot 6$ & 26 & $11 \cdot 9$ & 29 & $18 \cdot 0$ & 27 & \\
\hline $1-2 \mathrm{~d} /$ week & $65 \cdot 0$ & 330 & $59 \cdot 1$ & 68 & $64 \cdot 2$ & 156 & $70 \cdot 7$ & 106 & \\
\hline 3-4 d/week & 8.5 & 43 & $7 \cdot 0$ & 8 & $11 \cdot 1$ & 27 & $5 \cdot 3$ & 8 & \\
\hline $5-6 \mathrm{~d} /$ week & $3 \cdot 7$ & 19 & 3.5 & 4 & $4 \cdot 1$ & 10 & $3 \cdot 3$ & 5 & \\
\hline Every day & $6 \cdot 7$ & 34 & $7 \cdot 8$ & 9 & $8 \cdot 6$ & 21 & $2 \cdot 7$ & 4 & \\
\hline Dinner & - & 507 & - & 115 & - & 242 & - & 150 & 0.015 \\
\hline Never & $0 \cdot 8$ & 4 & 0.9 & 1 & 0.4 & 1 & $1 \cdot 3$ & 2 & \\
\hline $1-2 \mathrm{~d} /$ week & $6 \cdot 5$ & 33 & $8 \cdot 7$ & 10 & $3 \cdot 7$ & 9 & $9 \cdot 3$ & 14 & \\
\hline 3-4 d/week & $12 \cdot 6$ & 64 & $15 \cdot 7$ & 18 & $9 \cdot 9$ & 24 & $14 \cdot 7$ & 22 & \\
\hline 5-6 d/week & $31 \cdot 4$ & 159 & $27 \cdot 8$ & 32 & $29 \cdot 3$ & 71 & $37 \cdot 3$ & 56 & \\
\hline Every day & $48 \cdot 7$ & 247 & $47 \cdot 0$ & 54 & $56 \cdot 6$ & 137 & $37 \cdot 3$ & 56 & \\
\hline
\end{tabular}

CRF, Ca-rich food(s).

intakes. The FFQ used in the study only allowed children to indicate they consumed up to two or more cans of soda pop daily ${ }^{(30)}$. Another explanation for the lack of a relationship is that parental permissiveness may take some of the novelty out of such products for children. However, others have shown that low parental restriction rules were positively associated with soft drinks consumption among adolescents ${ }^{(39,40)}$.

Parental practices such as making milk available at meals and modelling milk intake were positively associated with $\mathrm{Ca}$ intake among children and adolescents in previous studies ${ }^{(4,5,7)}$. Results of the current study supported these findings, as $\mathrm{Ca}$ and dairy food intakes of Dedicated-Milk Providers/Drinkers and their offspring were greater than Sweet-Drink-Permissive Parents and Water Regulars and their offspring. These parents may have a strong affinity/commitment to drinking milk and a set of attitudes that result in their placing a high priority in making sure their diets are high in $\mathrm{Ca}$.

In the Water Regulars and Sweet-Drink Permissive Parents segments, barriers to more positive CRF practices may be concerns about cost and taste, as reported by others ${ }^{(6,41)}$. The Water Regulars group may have been more budget-driven, and encouraged drinking water as a way to make ends meet given that their educational attainment was lowest and use of food assistance programmes the highest compared with the other groups.
However, despite the parental emphasis on drinking water, their children reported the highest consumption of fruit drinks and soda pop of any segment, possibly based on greater availability in the home compared with other segments. Dairy/milk intolerance could be a barrier to dairy consumption, as more parents in the Water Regulars and Sweet-Drink-Permissive groups were Asian or Hispanic compared with Dedicated-Milk Providers/Drinkers, and these race/ethnic groups report a high level of perceived or real dairy/milk intolerance ${ }^{(19)}$.

Another way that the segmentation in the current study agreed with expectations was based on differences in the food environment. In a previous study, more frequent family meals were related to higher nutrient quality, including higher intakes of CRF among early adolescents $^{(42)}$. In the current study, the Sweet-Drink-Permissive Parents and Water Regulars segments reported consuming fewer family dinner meals than the Dedicated-Milk Providers/Drinkers. Data from a national US sample of adolescents showed that poverty rate was higher and maternal education was lower for those never eating family dinners compared with those eating family dinners more frequently ${ }^{(43)}$. The Sweet-Drink-Permissive Parents and Water Regulars in the current study were also more likely to use food assistance and were less educated compared with the Dedicated Milk Providers/Drinkers, indicating that education and income may influence 
frequency of family meals and the opportunity to consume milk with meals.

Limitations include the fact that the larger study ${ }^{(17)}$ of which the current study is part was not originally designed as a segmentation study; consequently, the segmentation variables may not represent a comprehensive list of parent attitudinal and behavioural variables that relate to $\mathrm{Ca}$ consumption of early adolescents. The resulting clusters may not be fully reflective of parent attitudes and behaviours, which could hinder the ability to generate richer insights into motivations and perceptions. A non-random convenience sample of well-educated Asian, Hispanic and non-Hispanic White parents was used, which limits application of results to a wider group of parents. All parents indicated that they could read/speak English and while a small proportion of Asian and Hispanic parents reported not speaking English at home, about $93 \%$ of these parents reported living in the USA for more than 10 years. A 2007 Pew Hispanic Center report indicated that length of residence in the USA was related to the ability of Hispanic immigrants to speak English well ${ }^{(44)}$. While a majority of foreign-born Hispanics (52\%) indicated they spoke only Spanish at home, only $28 \%$ said they spoke only Spanish on the job ${ }^{(44)}$ indicating that parents in the current study may have reported not speaking English at home because of preference rather than ability. Asian, Hispanic and non-Hispanic White parents and their early adolescent children were the subjects of the current study because these groups have been identified as being at highest risk of osteoporosis based on results from the National Osteoporosis Risk Assessment ${ }^{(45)}$.

In summary, the present study suggests that differences in $\mathrm{Ca}$ and beverage intakes among early adolescents are related to differences in parental CRF practices and perceptions regarding beverages. Therefore, nutrition educators may increase effectiveness of educational interventions by becoming aware of and working within existing parenting practices/perceptions.

\section{Acknowledgements}

This project was funded through money appropriated by Congress through the Hatch Act to the Agricultural Experiment Stations of land grant universities for multistate research projects. There are no conflicts of interest. M.R. conducted data analysis regarding differences across segments and wrote sections of the manuscript. D.D. designed and interpreted the cluster analysis, named and described the segments, and co-wrote the data analysis section. K.G. conducted the cluster analysis and co-wrote the data analysis section. G.A., M.B., C.Bruhn, M.C., S.M., B.O., S.W. and S.Z. developed measures used to collect data, collected data and wrote/reviewed sections of the manuscript. C.Boushey conducted data analysis to validate data collection measures, analysed $\mathrm{Ca}$ intake data, cleaned and prepared all data for analysis, collected data and wrote/reviewed sections of the manuscript. L.S.G. and C.G. wrote/reviewed sections of the manuscript.

\section{References}

1. Bailey RL, Dodd KW, Goldman JA et al. (2010) Estimation of total usual calcium and vitamin D intakes in the United States. J Nutr 140, 817-822.

2. Wang MC, Crawford PB, Hudes M et al. (2003) Diet in midpuberty and sedentary activity in prepuberty predict peak bone mass. Am J Clin Nutr 77, 495-503.

3. Kalkwarf HJ, Khoury JC \& Lanphear BP (2003) Milk intake during childhood and adolescence, adult bone density, and osteoporotic fractures in US women. Am J Clin Nutr 77, 257-265.

4. Neumark-Sztainer D, Hannan PJ, Story M et al. (2003) Family meal patterns: associations with sociodemographic characteristics and improved dietary intake among adolescents. J Am Diet Assoc 103, 317-322.

5. Larson NI, Story M, Wall M et al. (2006) Calcium and dairy intakes of adolescents are associated with their home environment, taste preferences, personal health beliefs, and meal patterns. J Am Diet Assoc 106, 1816-1824.

6. Winzenberg T, Hansen E \& Jones G (2008) How do women change osteoporosis-preventive behaviours in their children? Eur J Clin Nutr 62, 379-385.

7. Fisher JO, Mitchell DC, Smiciklas-Wright $\mathrm{H}$ et al. (2004) Meeting calcium recommendations during middle childhood reflects mother-daughter beverage choices and predicts bone mineral status. Am J Clin Nutr 79, 698-706.

8. Ohta H, Kuroda T, Onoe Y et al. (2010) Familial correlation of bone mineral density, birth data and lifestyle factors among adolescent daughters, mothers and grandmothers. J Bone Miner Metab 28, 690-695.

9. Beydoun MA \& Wang Y (2009) Parent-child dietary intake resemblance in the United States: evidence from a large representative survey. Soc Sci Med 68, 2137-2144.

10. Bandura A (1986) Social Foundations of Thought and Action: A Social Cognitive Theory. Englewood Cliffs, NJ: Prentice-Hall.

11. Sedlak CA, Doheny MO, Estok PJ et al. (2007) DXA, health beliefs, and osteoporosis prevention behaviors. I Aging Health 19, 742-756.

12. Cluskey M, Edlefsen M, Olson B et al. (2008) At home and away-from-home eating patterns influencing preadolescents' intake of calcium rich foods as perceived by Asian, Hispanic and non-Hispanic White parents. J Nutr Educ Behav 40, 72-79.

13. French MR, Vernace-Inserra F \& Hawker GA (2008) A prospective study to identify factors affecting adherence to recommended daily calcium intake in women with low bone mineral density. J Am Coll Nutr 27, 88-95.

14. French MR, Moore K, Vernace-Inserra F et al. (2005) Factors that influence adherence to calcium recommendations. Can J Diet Pract Res 66, 25-29.

15. Guthrie JF, Lin BH \& Frazao E (2002) Role of food prepared away from home in the American diet, 1977-78 versus 1994-96: changes and consequences. J Nutr Educ Behav 34, 140-150.

16. Devine CM, Jastran M, Jabs J et al. (2006) 'A lot of sacrifices': work-family spillover and the food choice coping strategies of low-wage employed parents. Soc Sci Med 63, 2591-2603.

17. Reicks M, Edlefsen M, Goodell LS et al. (2011) Individual and family correlates of calcium-rich food intake among 
parents of early adolescent children. J Am Diet Assoc 111, 376-384.

18. Satia-Abouta J, Patterson RE, Kristal AR et al. (2007) Psychosocial predictors of diet and acculturation in Chinese American and Chinese Canadian women. Ethn Health 7, 21-39.

19. Jackson KA \& Savaiano DA (2001) Lactose maldigestion, calcium intake and osteoporosis in African-, Asian-, and Hispanic-Americans. J Am Coll Nutr 20, 2 Suppl., 1985-207S.

20. Kroeze W, Werkman A \& Brug J (2006) A systematic review of randomized trials on the effectiveness of computertailored education on physical activity and dietary behaviors. Ann Behav Med 31, 205-223.

21. Eyles HC \& Mhurchu CN (2009) Does tailoring make a difference? A systematic review of the long-term effectiveness of tailored nutrition education for adults. Nutr Rev 67, 464-480.

22. Buckley M, Cowan C \& McCarthy M (2007) The convenience food market in Great Britain: convenience food lifestyle (CFL) segments. Appetite 49, 600-617.

23. Kolodinsky J \& Reynolds T (2009) Segmentation of overweight Americans and opportunities for social marketing. Int J Behav Nutr Phys Act 6, 13.

24. Sudo N, Degeneffe D, Vue H et al. (2009) Relationship between attitudes and indicators of obesity for midlife women. Health Educ Behav 36, 1082-1094.

25. Della LJ, DeJoy DM \& Lance CE (2008) Promoting fruit and vegetable consumption in different lifestyle groups: recommendations for program development based on behavioral research and consumer media data. Health Mark $Q$ 25, 66-96.

26. O'Connor TM, Hughes SO, Watson KB et al. (2010) Parenting practices are associated with fruit and vegetable consumption in pre-school children. Public Health Nutr 13, 91-101.

27. Byrd-Bredbenner C, Abbot JM \& Cussler E (2008) Mothers of young children cluster into 4 groups based on psychographic food decision influencers. Nutr Res 28, 506-516.

28. Cluskey M, Auld G, Edlefsen M et al. (2008) Calcium knowledge, concern and expectations for intake among parents of Asian, Hispanic and non-Hispanic white early adolescents. Forum for Family and Consumer Issues 13, http://ncsu.edu/ffci/publications/2008/v13-n3-2008-winter/ cluskey-auld-adlefsen-zaghloul-bock-bouskey-bruhn-goldbergnisner-olson-reicks.php

29. Edlefsen M, Reicks M, Goldberg DL et al. (2008) Strategies based on parental roles to influence intake of calcium-rich foods by Asian, Hispanic, and non-Hispanic white early adolescents. Prev Chronic Dis 5, http://www.cdc.gov/pcd/ issues/2008/oct/pdf/07_0174.pdf

30. Jensen JK, Gustafson D, Boushey CJ et al. (2004) Development of a food frequency questionnaire to estimate calcium intake of Asian, Hispanic, and White youth. J Am Diet Assoc 104, 762-769.
31. Yang J 2007. Use of duplicate plate method to evaluate dietary assessment methods. Dissertation, Purdue University; AAT 3296117.

32. Aldenderfer MS \& Blashfield RK (1984) Cluster Analysis. Newbury Park, CA: Sage Publications, Inc.

33. Degeneffe D, Kinsey J, Stinson T et al. (2009) Segmenting consumers for food defense communication strategies. Int $J$ Phys Distribution Logistics Manage 39, 365-403.

34. Matlik L, Savaiano D, McCabe G et al. (2007) Perceived milk intolerance is related to bone mineral content in 10- to 13-year-old adolescent females. Pediatrics 120, e669-e677.

35. National Cancer Institute 2010. Food Sources of Energy Among US Population, 2005-06. Risk Factor Monitoring and Methods Branch Website, Applied Research Program, National Cancer Institute. http://riskfactor.cancer.gov/diet/ foodsources/ (accessed August 2010).

36. Mrdjenovic G \& Levitsky DA (2003) Nutritional and energetic consequences of sweetened drink consumption in 6- to 13-year-old children. J Pediatr 142, 604-610.

37. Keller KL, Kirzner J, Pietrobelli A et al. (2009) Increased sweetened beverage intake is associated with reduced milk and calcium intake in 3- to 7-year-old children at multi-item laboratory lunches. J Am Diet Assoc 109, 497-501.

38. Fiorito LM, Marini M, Mitchell DC et al. (2010) Girls' early sweetened carbonated beverage intake predicts different patterns of beverage and nutrient intake across childhood and adolescence. J Am Diet Assoc 110, 543-550.

39. Verzeletti C, Maes L, Santinello $M$ et al. (2010) Soft drink consumption in adolescence: associations with foodrelated lifestyles and family rules in Belgium Flanders and the Veneto Region of Italy. Eur J Public Health 20, 312-317.

40. Nickelson J, Roseman MG \& Forthofer MS (2010) Associations between parental limits, school vending machine purchases, and soft drink consumption among Kentucky middle school students. J Nutr Educ Behav 42, 115-122.

41. Wood F, Robling M, Prout H et al. (2010) A question of balance: a qualitative study of mothers' interpretations of dietary recommendations. Ann Fam Med 8, 51-57.

42. Burgess-Champoux TL, Larson N, Neumark-Sztainer D et al. (2009) Are family meal patterns associated with overall diet quality during the transition from early to middle adolescence? J Nutr Educ Behav 41, 79-86.

43. Sen B (2006) Frequency of family dinner and adolescent body weight status: evidence from the national longitudinal survey of youth, 1997. Obesity (Silver Spring) 14, 2266-2276.

44. Hakimzadeh S \& Cohn D (2007) English Usage Among Hispanics in the United States. Washington, DC: Pew Hispanic Center.

45. Siris ES, Miller PD, Barrett-Connor E et al. (2001) Identification and fracture outcomes of undiagnosed low bone mineral density in postmenopausal women: results from the National Osteoporosis Risk Assessment. JAMA 286, 2815-2822. 